\title{
Semantics based biomedical knowledge search, integration and discovery
}

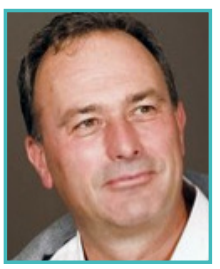

Barend Mons holds a chair in Biosemantics at the LUMC and is one of the scientific directors of NBIC. In addition he acts as a Life Sciences 'escience integrator' in the Netherlands escience centre. Currently, he coordinates the creation of the Data Integration and Stewardship Centre (DISC-ELIXIR) and in that capacity he is also the scientific representative of The Netherlands in the interim board of the ELIXIR ESFRI project.

Barend Mons is a molecular biologist by training and received his $\mathrm{PhD}$ on genetic differentiation of malaria parasites from Leiden University (1986). He performed over a decade of research on malaria genetics and vaccine development, also serving for 3 years the research department of the European Commission in this field. He did gain further experience in science management at the Research council of The Netherlands (NWO).

Barend is the co founder of three spin-off companies in biotechnological and semantic technologies. In 2000, he switched back to academia, focusing on the development of semantic technologies to manage big data and he founded the Biosemantics group.

His research is currently focused on nanopublications as a substrate for in silico knowledge discovery. Barend is also one of the founders of the Concept Web Alliance, with "nanopublications" as its first brainchild. Nanopublications are currently implemented in the semantic project of the Innovative Medicines Initiative (IMI) called Open PHACTS.

Barend Mons will talk about the role of semantic technologies and related standards applied to biomedical-molecular data integration and biomedical knowledge search and discovery.

He will challenge several established views in the field of the Semantic Web for Life Sciences, by also taking into account "data publishing" in a broad sense, including, e.g., biomedical communication, intellectual networking, and nanopublications, with an emphasis on the barriers to brake down in order to allow effective data exposure, sharing, searching, and integration, and to "in silico" discovery of new biomedical knowledge in the Big Data era.

This talk will introduce the need for a semantics based escience approach for "in silico" knowledge discovery. It will also show how such approach can indeed already support search, integration, and discovery. 